\title{
Performance Evaluation of Infrared and Visible Image Fusion Algorithms for Face Recognition
}

\author{
Jing Wang Jimin Liang Haihong Hu Yan Li Bin Feng \\ Life Science Research Center, Xidian University, Xi’an 710071, P. R. China
}

\begin{abstract}
Fusion of infrared and visible image is a potential solution to improve face recognition performance. In this paper, we propose a new fusion method based on singular value decomposition (SVD) and apply it to multiple spectrum face recognition. The performance of the proposed SVD-based fusion method is compared with that of average fusion, Laplacian Pyramid Decomposition (LPD) based fusion and Discrete Wavelet Transform (DWT) based fusion methods. The performances of the four image fusion methods for face recognition are analyzed by statistical experiments and the results show that the SVD-based fusion method is better in most conditions.
\end{abstract}

Keywords: Image fusion, Singular value decomposition (SVD), Performance evaluation, Face recognition

\section{Introduction}

Human face recognition is a challenging task and has many applications, such as security systems, defense applications, and intelligent machines. Recognition based on the visual image is sensitive to variations in illumination conditions [1]. Recognition of faces using different imaging modalities, in particular infrared (IR) images has become an area of growing interest [2]-[3]. Thermal IR imagery is nearly invariant to changes in ambient illumination, and provides a capability for identification under all lighting conditions including total darkness [4]. Despite its advantages, IR imagery has some limitations including that it is opaque to glass. Objects made of glass completely hiding the face parts located behind them. These can degrade face recognition accuracy on thermal IR face image. To address these serious limitations of visual spectrum and IR, fusing IR with visible imagery is a potential solution to improve face recognition performance. In this paper, the multiple spectrum face database of Equinox Corporation is used for numerical experiments and two classical algorithms, PCA and LDA, are adopted for face recognition.
In recent years, a number of image fusion methods have been proposed. The most common procedures are methods based on simple averaging, Lap1acian pyramid method [18] and wavelet transform method [20]. In this paper, a new multimodal image fusion method based on singular value decomposition (SVD) is proposed and applied to multiple spectrum face recognition problem. Its performance is compared with that of other three fusion methods - Average fusion, Laplacian pyramid decomposition (LPD) based, and discrete wavelet transform (DWT) based fusion methods.

The objective evaluation of image fusion method is very difficult if the reference image is not available. Such that the subjective evaluation is often applied. Regarding the object detection and recognition applications, the performance of detection and recognition can be used as final evaluation index for fusion methods. In this paper, we use statistical face recognition performance to evaluate the fusion methods. Following the evaluation of biometric algorithms [13]-[15], randomized experiments are performed for all tests. Each test randomly assemble probe and gallery sets. A sufficient large number of runs are conducted to generate statistical evaluation results, such as, CMC with error bars, the table of Rank 1 statistical recognition performance for all tests and the table of pairwise comparisons based upon the difference in recognition rate statistic.

\section{Previous work}

Socolinsky et al. [6] shows results on the use of thermal infrared and visible imagery for face recognition in operational scenarios. The statistical significance of their analysis is based on their randomized approach to select gallery and probe images for experiments with three different algorithms: PCA with Mahalanobis angle distance, LDA with angle distance and the (blinded for review) algorithm. Heo et al. [9] considered two types of visible and IR fusion, the first at the data level and the second at the decision level. To deal with occlusions caused by eyeglasses, they used ellipse fitting to detect the 
eyeglasses regions in the IR image and replaced them with an eye template. Using a commercial face recognition system (i.e. FaceIt), they demonstrated improvements in recognition accuracy. Gyaourova et al. [10] presented a fusion method for combining IR and visible light images for the purposes of face recognition. Their fusion scheme is pixel-based, operates in the wavelet domain, and employs genetic algorithms (GAs) to decide how to combine IR with visible information. The algorithm aims at improved and robust recognition performance across variable lighting, facial expression, and presences of eyeglasses. Their experimental results show substantial improvements recognition performance overall. Bebis et al. [11] presented and compared two different fusion schemes for combining IR and visible imagery for the purpose of face recognition. Two different fusion schemes had been investigated in this paper. The first scheme was pixel-based, operating in the wavelet domain. The second scheme was feature-based, operating in the eigenspace domain. Both schemes aimed at improved and robust recognition performance across variable lighting and presence/absence of eyeglasses.

The aforementioned works conduct experiments on fixed gallery and probe databases such that their results lack of statistical meanings. In this paper, we take gallery and probe sub-databases randomly from the entire database and conduct a large number of test for each testing condition to generate statistically evaluation results. Although Socolinsky et al. [6] also performed random experiments, they used only one kind of fusion method. In this paper, we evaluate four kinds of fusion methods for face recognition.

\section{Image fusion methods}

\subsection{LPD fusion and DWT fusion}

Both the LPD [18] and DWT [20] based fusion methods belong to the multiscale decomposition fusion methods. They differ in the way of multiscale decomposition and the fusion rules. The multiscale decomposition image fusion method usually runs in three steps. Firstly, the original calibrated images are decomposed based on some specific multiscale decomposition method. Secondly, the two multiscale image sequences are fused into one multiscale image sequence according with certain fusion strategy. Finally, the fusion image is generated by reconstructing the fused multiscale image sequence.

\subsection{SVD Fusion}

The singular value decomposition (SVD) is widely used in data compression, signal processing, pattern recognition, etc [23]-[24]. The SVD of a matrix $A$ is defined as [25]:

$$
A=U S V^{T}=\sum_{i=1}^{r} \lambda_{i}^{\frac{1}{2}} \mu_{i} v_{i}^{T}
$$

where $\quad U=\left[\mu_{1}, \mu_{2}, \cdots, \mu_{m}\right] \in R^{m \times m} \quad$ and $V=\left[v_{1}, v_{2}, \cdots, v_{n}\right] \in R^{n \times n}$ are orthogonal. The matrix $S=\operatorname{diag}\left[\lambda_{1}^{\frac{1}{2}}, \lambda_{2}^{\frac{1}{2}}, \cdots, \lambda_{r}^{\frac{1}{2}}, 0, \cdots, 0\right] \in R^{m \times n}$ and $\lambda_{1}^{\frac{1}{2}} \geq \lambda_{2}^{\frac{1}{2}} \geq \cdots \geq \lambda_{r}^{\frac{1}{2}} \geq 0$.The number $\left\{\lambda_{i}\right\}$ are the eigenvalues of $A A^{T}$ and $A^{T} A$. The columns of $U$ are the eigenvectors of $A A^{T}$ and the columns of $V$ are eigenvectors of $A^{T} A$.

Any grayscale image can be considered as a two dimensional matrix, thus can be decomposed using SVD. After decomposition, the image is sliced into $r$ levels with each slice equals to $\lambda_{i}^{\frac{1}{2}} \mu_{i} v_{i}^{T}$. In equation (1), the norm of each slice equals:

$$
\begin{aligned}
\left\|\lambda_{i}^{\frac{1}{2}} \mu_{i} v_{i}^{T}\right\| & =\lambda_{i}\left\|\mu_{i} v_{i}^{T}\right\|=\lambda_{i} \operatorname{tr}\left[\left(\mu_{i} v_{i}^{T}\right)^{T}\left(\mu_{i} v_{i}^{T}\right)\right] \\
& =\lambda_{i} \operatorname{tr}\left[v_{i} \mu_{i}^{T} \mu_{i} v_{i}^{T}\right]=\lambda_{i} \operatorname{tr}\left[v_{i} v_{i}^{T}\right] \\
& =\lambda_{i}\left(v_{i}^{T} v_{i}\right)=\lambda_{i} .
\end{aligned}
$$

The norm of an image matrix can be regarded as the image energy. Equation 2 suggests that the image energy of the ith slice is equal to its corresponding eigenvalue of this level.

In this paper, the face images are decomposed into three layers according to the energy distribution. So a grayscale image $g$ can be decomposed into the following form:

$$
g=g^{L}+g^{H}+g^{N}
$$

$$
\begin{aligned}
\text { where } \quad g & =\sum_{i=1}^{r} \lambda_{i}^{\frac{1}{2}} \mu_{i} v_{i}^{T}, \quad g^{L}=\sum_{i=I_{l}}^{I_{h}-1} \lambda_{i}^{\frac{1}{2}} \mu_{i} v_{i}^{T}, \\
g^{H} & =\sum_{i=I_{h}}^{I_{n}-1} \lambda_{i}^{\frac{1}{2}} \mu_{i} v_{i}^{T}, \quad g^{N}=\sum_{i=I_{n}}^{r} \lambda_{i}^{\frac{1}{2}} \mu_{i} v_{i}^{T},
\end{aligned}
$$

$I_{l}=1$, and $I_{l} \leq I_{h} \leq I_{n} \leq r$. It is easy to prove that the following equation holds: 


$$
\left\|g^{L, H, N}\right\|=\sum_{i=I_{l}, I_{h}, I_{n}}^{I_{h}, I_{n}, r-1} \lambda_{i} .
$$

By selecting the value of $I_{h}$ and $I_{n}$, a grayscale image can be decomposed into three layers with different energy resolution. With the eigenvalues arranged in descending order, $g^{L}$ consists of image slices with greater eigenvalues, $g^{H}$ consists of image slices with medium eigenvalues, and $g^{N}$ level consists of image levels with smaller eigenvalues. As a result, the low resolution $g^{L}$ is of greater energy percentage, the high resolution $g^{H}$ of medium energy percentage, and the ultrahigh resolution $g^{N}$ of smaller energy percentage. In the following experiments, we make the low, high and ultrahigh resolution images account for 99, 0.99, and 0.01 percent of the total image energy.

For image fusion problem, the original image $g_{1}$ and $g_{2}$ are sliced with the same percentage of image energy using the method described above. The results are denoted as $g_{1}^{L}, g_{1}^{H}, g_{1}^{N}$, and $g_{2}^{L}, g_{2}^{H}, g_{2}^{N}$, respectively. Each pair of the corresponding layers has the same energy. Our fusion algorithm fuse $g_{1}^{L}$ and $g_{2}^{L}$ into a low resolution layer denoted as $f^{L}$, and fuse $g_{1}^{H}$ and $g_{2}^{H}$ into a high resolution layer denoted as $f^{H}$. The ultrahigh resolution layers can be approximately considered as image noise and be neglected. Finally, the $f^{L}$ and $f^{H}$ are used to reconstruct the fusion result, which denoted as $f$.

In this paper, for the LPD, DWT and SVD fusion methods, we discard the visible light low resolution layers and only use IR low resolution layers to reconstruct the fusion image. For the high resolution layers, we use the fusion scheme proposed by Burt [22].

\section{Performance evaluation}

We evaluate the infrared and visible image fusion methods by statistical face recognition performance. Following the evaluation of biometric algorithms [14][15] and the CSU software [13], we perform random experiments for all tests. Each test takes random permutations of the probe and gallery sets for each subject. It needs a list of subjects with at least three replicate images per subject. We use three replicates per subject. In the experiments, we first select at random one of the three images for each subject to serve as a probe image and one of the remaining two to serve as the gallery image, and then compute recognition rates. The nearest neighborhood classifier (NNC) is adopted for recognition.

After a sufficient large number of random experiments, we can get the distribution of recognition rates. The distribution of these recognition rates represents a good approximation to the probability distribution for the larger population of possible probe and gallery images. Some statistical measurements are defined in [13]. (1) Mode. The peak at recognition count on a certain rank. (2) Mean. It is a weighted average recognition rate at each rank. (3) two-sided confidence interval (lower, upper). This is done by identifying the central portion of each distribution accounting for 95 percent of the outcomes.

Besides computing the recognition rate on a certain modality (e.g. IR images), we also compare the recognition rate on two different modalities (e.g. Vi images and IR images) according to the method in [13]. For each of the 10000 trials, since a predominance of positive values indicates that the performance on this modality is better, a predominance of negative values indicates that the performance on the other modality is better, and a distribution centered around zero indicated there is no difference. After finishing the experiment, we can get the probability of the recognition rate on one kind of modality is greater (or smaller) than the other.

After a large number of random experiments, we compute the mode, mean recognition rate and confidence intervals at each rank. Three statistical results are generated: (1) the cumulative match curves with 95 percent confidence error bars; (2) the table of Rank 1 statistical recognition performance for all tests; (3) the table of pairwise comparisons based upon the difference in recognition rate statistic.

\section{Experiment}

\subsection{Face Dataset}

We use the face database collected by Equinox Corporation under DARPA's Human ID program [17]. To avoid the image registration problem, we only use the long-wave infrared (LWIR) and the corresponding visible light images from this database. Each pair of LWIR and visible light images were taken simultaneously and co-registered with $1 / 3$ pixel accuracy. Some example images are given in Fig. 1. 
The LWIR images were stored as grayscale images with 12 bits per pixels and original 12 bits grayscale LWIR images were converted into 8 bits before experiment. The visible images are also grayscale images represented with 8 bits per pixel. The database used for the evaluation of face recognition performance is shown in Table1. The database consists of visual and thermal IR images of 2,106 (1053 per modality) face images from 89 individuals. Images taken with frontal lighting conditions are used as the train images. Test images are divided into different conditions. Test 1 to Test 3 respectively tests the effect of illumination, expression, glasses on recognition performance. Test4 tests the three conditions on recognition performance together.

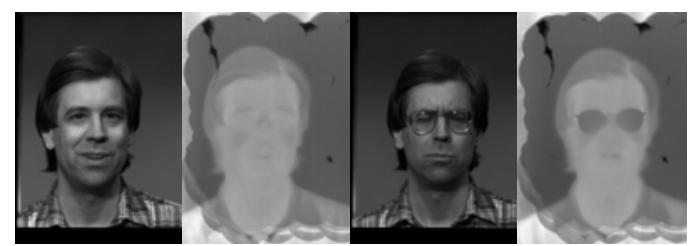

Fig. 1: Examples of visual and thermal images.

\begin{tabular}{|c|c|c|c|c|}
\hline dataset & $\begin{array}{c}\text { Number } \\
\text { of } \\
\text { visible/IR }\end{array}$ & glasses & illumination & expression \\
\hline train & $267 / 267$ & off & frontal & neutral \\
\hline Test1 & $264 / 264$ & off & $\begin{array}{c}\text { frontal } \\
\text { right and } \\
\text { left }\end{array}$ & neutral \\
\hline Test2 & $258 / 258$ & off & frontal & vary \\
\hline Test3 & $135 / 135$ & on & frontal & neutral \\
\hline Test4 & $129 / 129$ & $\begin{array}{c}\text { on and } \\
\text { off }\end{array}$ & $\begin{array}{c}\text { frontal } \\
\text { right and } \\
\text { left }\end{array}$ & vary \\
\hline
\end{tabular}

Table 1: Experiment database description.

\subsection{Normalization}

Eye coordinates for visible and thermal images, were manually located from visible images. This is one of the challenges of using only thermal images as it is very hard to determine where the eyes are and thus the use of a co-registered camera which acquires both visual and thermal images is necessary to proper face normalization for scale. After alignment, the image is cropped by using an elliptical mask and image borders such that only the face from forehead to chin and cheek to cheek is visible. We follow the convention in the CSU software [13] and use $130 \times 150$ resolution versions of the original visible and IR images in creating the face space. Fig. 2 shows examples of normalized faces acquired from both modalities.

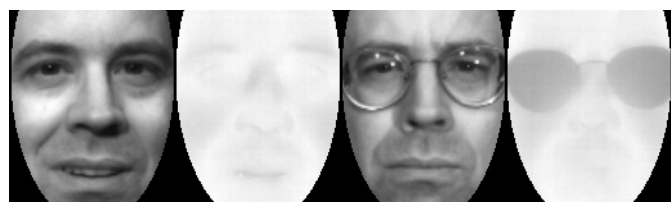

Fig. 2: Examples of normalized faces (from left to right: visual, infrared, visual with glasses, infrared with glasses).

\subsection{Structure of the Experiment}

We perform experiments with six different kinds of face images, two different face recognition algorithms and three different test conditions. The six kinds of face images are visible images, IR images, average fusion images, LPD based fusion images, DWT based fusion images and SVD based fusion images. The two algorithms are PCA and LDA, which are standard algorithms which widely available in the literature. Three test conditions are illumination, expression and glasses. The purpose of this experiment is to compare face recognition performance on different fusion images under different conditions.

\subsection{Experimental Results and Discussion}

We perform all experiments described above. Fig. 3Fig. 6 shows the CMC with 95\% confidence error bars for all experiments. A summary of top match recognition performance is shown in Table2. Table3 displays the pairwise comparisons results for the six kinds of face images.

Fig. 3-Fig. 5 denotes the recognition performance under illumination, expression, glasses respectively and Fig. 6 denotes the recognition result under the three conditions together. Fig (a) in each figure expresses the results using PCA, and Fig (b) in each figure shows the results using LDA.

Fig.3-Fig.6 demonstrates the first 10 best matches of different recognition methods in terms of matching scores. As shown in Fig.3-Fig.6, the recognition rate with fusion images is better than with visible and IR images alone in most cases. For every CMC, the top three curves are extremely close, that's shows that the recognition performance on SVD based fusion images is very similar with LPD and DWT based fusion images. Fig.3-Fig.6 also indicates that the recognition performance using LDA algorithm is better than using PCA algorithm under all kinds of conditions. In addition, from the $95 \%$ confidence error bars on these curves, we know that the recognition performance under illumination tests is the most stable for all images, and it under glasses tests is the most unsteady. 


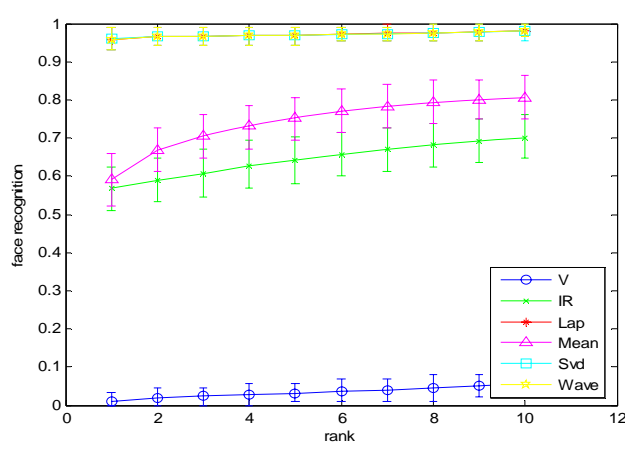

(a)

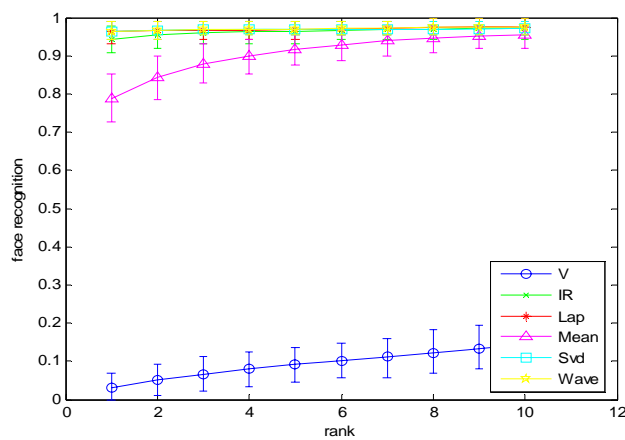

(b)

Fig. 3: CMC with 95\% confidence error bars for illumination testing on all kinds of images. (a )using PCA.(b)using LDA.

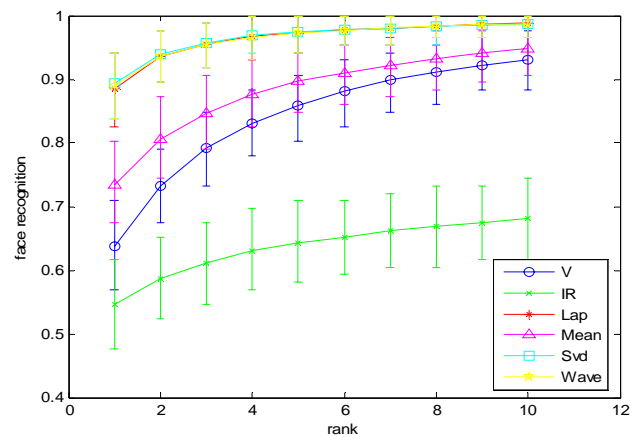

(a)

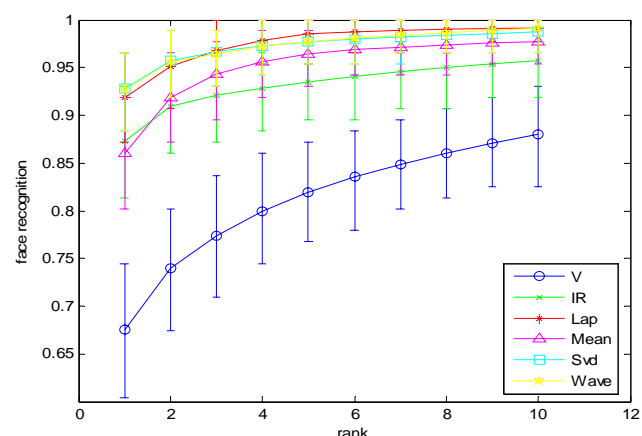

(b)

Fig. 4: CMC with 95\% confidence error bars for expression testing on all kinds of images. (a)using PCA (b)using LDA.

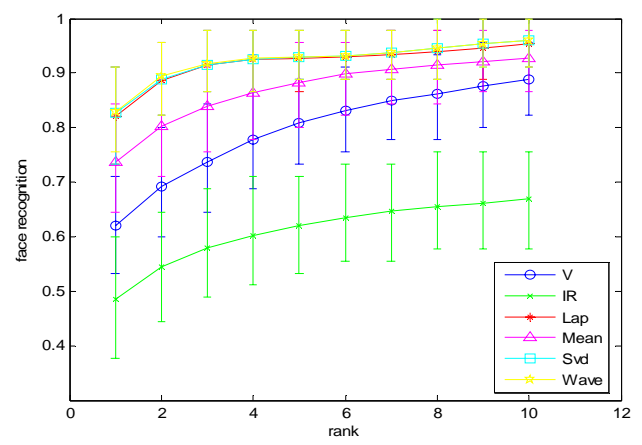

(a)

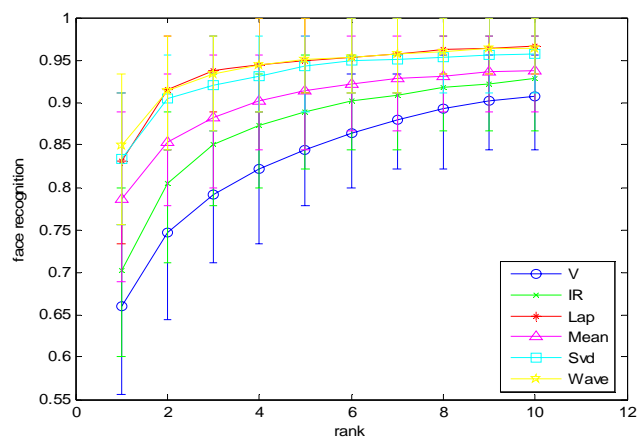

(b)

Fig. 5: CMC with 95\% confidence error bars for glasses testing on all kinds of images. (a)using PCA (b)using LDA.

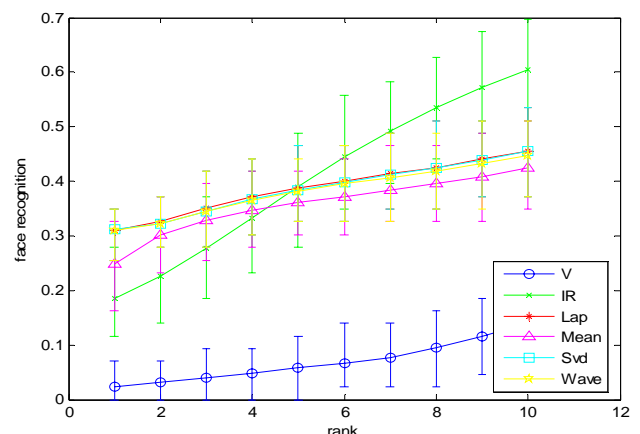

(a)

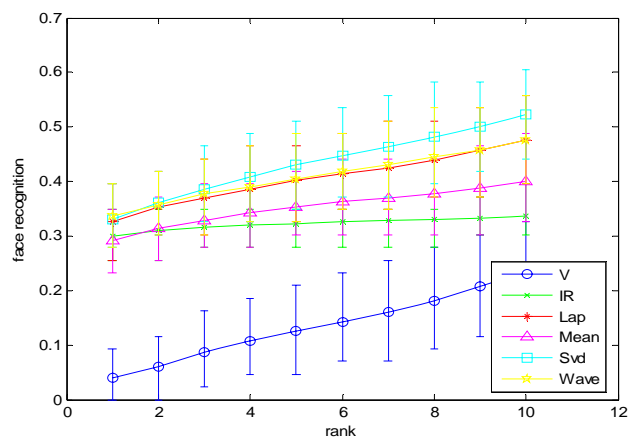

(b)

Fig. 6: CMC curves with 95\% confidence error bars for all conditions testing on all kinds of images. (a) using PCA. (b) using LDA. 


\begin{tabular}{|c|c|c|c|c|c|c|c|c|}
\hline \multirow[t]{2}{*}{ Images } & \multicolumn{4}{|c|}{ PCA } & \multicolumn{4}{|c|}{ LDA } \\
\hline & Mode\% & Mean\% & Upper\% & Lower\% & Mode\% & Mean\% & Upper\% & Lower\% \\
\hline \multirow{4}{*}{$\mathrm{Vi}$} & $1.1 \%$ & $1.0 \%$ & $3.4 \%$ & $0.0 \%$ & $2.3 \%$ & $3.2 \%$ & $6.8 \%$ & $0.0 \%$ \\
\hline & $64.0 \%$ & $63.9 \%$ & $70.9 \%$ & $57.0 \%$ & $68.6 \%$ & $67.6 \%$ & $74.4 \%$ & $60.5 \%$ \\
\hline & $62.2 \%$ & $62.0 \%$ & $71.1 \%$ & $53.3 \%$ & $64.4 \%$ & $66.1 \%$ & $75.6 \%$ & $55.6 \%$ \\
\hline & $2.3 \%$ & $2.4 \%$ & $7.0 \%$ & $0.0 \%$ & $2.3 \%$ & $3.9 \%$ & $9.3 \%$ & $0.0 \%$ \\
\hline \multirow{4}{*}{ IR } & $56.8 \%$ & $56.8 \%$ & $62.5 \%$ & $51.1 \%$ & $94.3 \%$ & $94.3 \%$ & $97.7 \%$ & $90.9 \%$ \\
\hline & $54.7 \%$ & $54.6 \%$ & $61.6 \%$ & $47.7 \%$ & $87.2 \%$ & $87.3 \%$ & $93.0 \%$ & $81.4 \%$ \\
\hline & $48.9 \%$ & $48.4 \%$ & $60.0 \%$ & $37.8 \%$ & $71.1 \%$ & $70.3 \%$ & $80.0 \%$ & $60.0 \%$ \\
\hline & $18.6 \%$ & $18.6 \%$ & $27.9 \%$ & $11.6 \%$ & $30.2 \%$ & $30.1 \%$ & $34.9 \%$ & $25.6 \%$ \\
\hline \multirow{4}{*}{ Average } & $59.1 \%$ & $59.1 \%$ & $65.9 \%$ & $52.3 \%$ & $78.4 \%$ & $78.8 \%$ & $85.2 \%$ & $72.7 \%$ \\
\hline & $73.3 \%$ & $73.5 \%$ & $80.2 \%$ & $67.4 \%$ & $86.0 \%$ & $86.0 \%$ & $91.9 \%$ & $80.2 \%$ \\
\hline & $73.3 \%$ & $73.8 \%$ & $84.4 \%$ & $64.4 \%$ & $77.8 \%$ & $78.7 \%$ & $88.9 \%$ & $68.9 \%$ \\
\hline & $25.6 \%$ & $24.8 \%$ & $32.6 \%$ & $16.3 \%$ & $30.2 \%$ & $29.0 \%$ & $34.9 \%$ & $23.3 \%$ \\
\hline \multirow{4}{*}{ LPD } & $95.5 \%$ & $95.8 \%$ & $98.9 \%$ & $93.2 \%$ & $96.6 \%$ & $96.3 \%$ & $98.9 \%$ & $93.2 \%$ \\
\hline & $88.4 \%$ & $88.5 \%$ & $94.2 \%$ & $82.6 \%$ & $91.9 \%$ & $91.9 \%$ & $96.5 \%$ & $87.2 \%$ \\
\hline & $82.2 \%$ & $82.0 \%$ & $91.1 \%$ & $73.3 \%$ & $82.2 \%$ & $83.1 \%$ & $91.1 \%$ & $73.3 \%$ \\
\hline & $30.2 \%$ & $30.8 \%$ & $34.9 \%$ & $25.6 \%$ & $32.6 \%$ & $32.5 \%$ & $39.5 \%$ & $25.6 \%$ \\
\hline \multirow{4}{*}{ DWT } & $95.5 \%$ & $95.8 \%$ & $98.9 \%$ & $93.2 \%$ & $96.6 \%$ & $96.4 \%$ & $98.9 \%$ & $94.3 \%$ \\
\hline & $89.5 \%$ & $89.1 \%$ & $94.2 \%$ & $83.7 \%$ & $93.0 \%$ & $92.7 \%$ & $96.5 \%$ & $88.4 \%$ \\
\hline & $82.2 \%$ & $83.0 \%$ & $91.1 \%$ & $75.6 \%$ & $84.4 \%$ & $85.0 \%$ & $93.3 \%$ & $75.6 \%$ \\
\hline & $30.2 \%$ & $30.8 \%$ & $34.9 \%$ & $25.6 \%$ & $34.9 \%$ & $33.8 \%$ & $39.5 \%$ & $27.9 \%$ \\
\hline \multirow{4}{*}{ SVD } & $95.5 \%$ & $95.9 \%$ & $98.9 \%$ & $93.2 \%$ & $96.6 \%$ & $96.3 \%$ & $98.9 \%$ & $94.3 \%$ \\
\hline & $89.5 \%$ & $89.4 \%$ & $94.2 \%$ & $83.7 \%$ & $93.0 \%$ & $92.8 \%$ & $96.5 \%$ & $88.4 \%$ \\
\hline & $82.2 \%$ & $82.7 \%$ & $91.1 \%$ & $73.3 \%$ & $82.2 \%$ & $83.3 \%$ & $91.1 \%$ & $75.6 \%$ \\
\hline & $32.6 \%$ & $31.1 \%$ & $34.9 \%$ & $25.6 \%$ & $32.6 \%$ & $33.1 \%$ & $39.5 \%$ & $27.9 \%$ \\
\hline
\end{tabular}

Table 2: Rank 1 correct match percentage for all tests of permutation results (The first entry in each cell shows the performance measured under different illumination conditions, the second entry under different expression conditions, the third entry under glasses conditions, and the bottom entry under all conditions).

\begin{tabular}{|c|c|c|c|c|c|c|c|c|c|}
\hline \multirow{3}{*}{$\begin{array}{l}\text { Images1 } \\
\mathrm{Vi}\end{array}$} & \multirow{3}{*}{$\begin{array}{l}\text { Images2 } \\
\text { IR }\end{array}$} & \multicolumn{4}{|c|}{ Probability of Recognition Rate $1>2$} & \multicolumn{4}{|c|}{ Probability of Recognition Rate $1<2$} \\
\hline & & 0.0000 & 0.9685 & 0.9512 & 0.0000 & 1.0000 & 0.0190 & 0.0252 & 0.9998 \\
\hline & & 0.0000 & 0.0000 & 0.2128 & 0.0000 & 1.0000 & 1.0000 & 0.6795 & 1.0000 \\
\hline \multirow[t]{2}{*}{ Vi } & Average & 0.0000 & 0.0039 & 0.0036 & 0.0000 & 1.0000 & 0.9904 & 0.9825 & 1.0000 \\
\hline & & 0.0000 & 0.0000 & 0.0081 & 0.0000 & 1.0000 & 1.0000 & 0.9763 & 1.0000 \\
\hline \multirow[t]{2}{*}{$\mathrm{Vi}$} & LPD & 0.0000 & 0.0000 & 0.0000 & 0.0000 & 1.0000 & 1.0000 & 0.9998 & 1.0000 \\
\hline & & 0.0000 & 0.0000 & 0.0009 & 0.0000 & 1.0000 & 1.0000 & 0.9967 & 1.0000 \\
\hline \multirow[t]{2}{*}{$\mathrm{Vi}$} & DWT & 0.0000 & 0.0000 & 0.0000 & 0.0000 & 1.0000 & 1.0000 & 0.9999 & 1.0000 \\
\hline & & 0.0000 & 0.0000 & 0.0000 & 0.0000 & 1.0000 & 1.0000 & 0.9992 & 1.0000 \\
\hline \multirow[t]{2}{*}{$\mathrm{Vi}$} & SVD & 0.0000 & 0.0000 & 0.0000 & 0.0000 & 1.0000 & 1.0000 & 0.9999 & 1.0000 \\
\hline & & 0.0000 & 0.0000 & 0.0000 & 0.0000 & 1.0000 & 1.0000 & 0.9993 & 1.0000 \\
\hline \multirow[t]{2}{*}{ IR } & Average & 0.2601 & 0.0000 & 0.0002 & 0.0695 & 0.6504 & 1.0000 & 0.9995 & 0.8454 \\
\hline & & 1.0000 & 0.5806 & 0.0438 & 0.4911 & 0.0000 & 0.3006 & 0.9035 & 0.1865 \\
\hline \multirow[t]{2}{*}{ IR } & LPD & 0.0000 & 0.0000 & 0.0000 & 0.0015 & 1.0000 & 1.0000 & 1.0000 & 0.9935 \\
\hline & & 0.0760 & 0.0482 & 0.0053 & 0.1081 & 0.7778 & 0.8979 & 0.9847 & 0.6485 \\
\hline \multirow[t]{2}{*}{ IR } & DWT & 0.0000 & 0.0000 & 0.0000 & 0.0013 & 1.0000 & 1.0000 & 1.0000 & 0.9942 \\
\hline & & 0.0807 & 0.0162 & 0.0303 & 0.0328 & 0.7781 & 0.9560 & 0.6455 & 0.7984 \\
\hline \multirow[t]{2}{*}{ IR } & SVD & 0.0000 & 0.0000 & 0.0000 & 0.0008 & 1.0000 & 1.0000 & 1.0000 & 0.9962 \\
\hline & & 0.0651 & 0.0217 & 0.0149 & 0.0418 & 0.7897 & 0.9486 & 0.8117 & 0.7295 \\
\hline \multirow[t]{2}{*}{ LPD } & Average & 1.0000 & 1.0000 & 0.9603 & 0.8926 & 0.0000 & 0.0000 & 0.0078 & 0.0310 \\
\hline & & 1.0000 & 0.9662 & 0.7717 & 0.7753 & 0.0000 & 0.0119 & 0.1047 & 0.0515 \\
\hline \multirow[t]{2}{*}{ LPD } & DWT & 0.0000 & 0.1777 & 0.1587 & 0.0375 & 0.0209 & 0.4638 & 0.4452 & 0.0433 \\
\hline & & 0.1341 & 0.2131 & 0.1133 & 0.1167 & 0.1842 & 0.5532 & 04255 & 0.4908 \\
\hline \multirow[t]{2}{*}{ LPD } & SVD & 0.0544 & 0.1518 & 0.1887 & 0.0381 & 0.1167 & 0.5700 & 0.3855 & 0.1390 \\
\hline & & 0.2160 & 0.2374 & 0.0903 & 0.2043 & 0.2409 & 0.5621 & 0.6580 & 0.3871 \\
\hline \multirow[t]{2}{*}{ SVD } & Average & 1.0000 & 1.0000 & 0.9736 & 0.9035 & 0.0000 & 0.0000 & 0.0055 & 0.0258 \\
\hline & & 1.0000 & 0.9842 & 0.9993 & 0.8398 & 0.0000 & 0.0052 & 0.0001 & 0.0194 \\
\hline \multirow[t]{2}{*}{ SVD } & DWT & 0.1150 & 0.4086 & 0.0801 & 0.1140 & 0.0724 & 0.2240 & 0.2021 & 0.0215 \\
\hline & & 0.2226 & 0.3993 & 0.5214 & 0.1608 & 0.2492 & 0.3671 & 0.1345 & 0.3655 \\
\hline
\end{tabular}

Table 3: Pairwise comparisons based upon the difference in recognition rate statistic (The top and bottom row in each cell respectively shows the results using PCA and LDA. In each row (PCA/LDA), In each row (PCA/LDA), the first line shows the comparison results under illumination tests, the second line under expression tests, the fourth line under all test). 
Table 2 shows the mode, mean, minimum and maximum performance for all images under different conditions at rank 1 . This table indicates the statistical results clearly. From the results using PCA, we can conclude that all fusion images improve the performance largely over the single spectrum image. However, when using LDA algorithm, the performance on fusion images is not always better than visible images and IR images. Under illumination and expression tests, the performance on IR images is better than Average fusion images, but worse than that of the other three fusion images. In a word, whether using PCA algorithm or LDA algorithm, the recognition performance on SVD based fusion images, LPD based fusion images and DWT based fusion images is always better than using visible and IR images. Among the fusion images, the recognition performance on SVD based fusion images is very similar with the LPD and DWT based fusion images, sometimes is a little better.

Table 3 shows the pairwise comparisons results on the probability of recognition rate on modality 1 greater (or smaller) than that of on modality2. From this table, we can see that: (1)The performance on SVD based fusion and DWT based fusion images has a greater probability better than LPD based fusion images. (2)The performance on SVD based images has a greater probability better than DWT based fusion images most of conditions.

The experiment results show that the recognition performance of SVD based fusion images is better than DWT and LPD based fusion images in most conditions. It advantage may comes form that the SVD based fusion method does not need subsampling and interpolation operations, thus avoids the drawback of introducing high frequency artifacts into the fusion images.

\section{Conclusion}

In this paper, we evaluate the fusion images by statistical face recognition performance. All the experiments are did according to the CSU software [13] on using permutation probe-gallery, and some statistical results are generated, e.g. CMC with error bars, the table of Rank 1 statistical recognition performance for all tests and the table of pairwise comparisons based upon the difference in recognition rate statistic. These three statistic results have their respective virtues, and the results got from them are similar. Since every test did many times and selected the gallery and probe sets randomly, the results are more believable.

We propose a SVD based fusion method and apply it to multiple spectrum face recognition. Its performance is compared with that of other three classical image fusion methods. The experiment results indicate that the SVD based fusion method heightens the face recognition performance greatly. The experiment results also show that the recognition performances of SVD, LPD and DWT based fusion methods are very much alike, and that the SVD based fusion method is a little better in most conditions. Although the SVD based fusion method improves the recognition performance under all tests, applicability still needs more investigation.

\section{Acknowledgement}

This work is partially supported by the Natural Science Foundation of China (Grant No. 60402038), the Chair Professors of the Cheung Kong Scholars, the Program for Cheung Kong Scholars and Innovative Research Team in University (PCSIRT), and Natural Science Foundation of Shaanxi (Grant No. 2005F35).

\section{References}

[1] Y. Adini, Y. Moses and S. Ullman, Face recognition: the problem of compensating for changes in illumination direction. IEEE Transactions on Pattern Analysis and Machine Intelligence. 19:721-732, 1997.

[2] Y. Yoshitomi, T. Miyaura, S. Tomita and S. Kimura, Face identification using thermal image processing. Proceedings. 6th IEEE International Workshop on Robot and Human Communication, pp. 374-379, 1997

[3] D. Socolinsky, A. Selinger and J. Neuheisel, Face recognition with visible and thermal infrared imagery. Computer Vision and Image Understanding, 91:72-114, 2003.

[4] G. Friedrich and Y. Yeshurun, Seeing people in the dark: face recognition in infrared images. 2nd Biologically Motivated Computer Vision, pp. 348359, 2002.

[5] S. Kong, J. Heo, B. Abidi, J. Paik, and M. Abidi, Recent Advances in Visual and Infrared Face Recognition - A Review. The Journal of Computer Vision and Image Understanding, 97:103-135, 2005.

[6] D. Socolinsky and A. Selinger, Thermal face recognition in an operational scenario. In: IEEE Conference on Computer Vision and Pattern Recognition (CVPR), 2004.

[7] A. Selinger and D. Socolinsky, Appearance-based facial recognition using visible and thermal imagery: A comparative study. Technical report, Equinox Corporation.02-01, 2002. 
[8] X. Chen, P. Flynn and K. Bowyer, Pca-based face recognition in infrared imagery: Baseline and comparative studies. In: IEEE International Workshop on Analysis and Modeling of Faces and Gestures, Nice, France, pp. 127-134, 2003.

[9] J. Heo, S. Kong, B. Abidi and M. Abidi, Fusion of visual and thermal signatures with eyeglass removal for robust face recognition. In: IEEE International Workshop on Object Tracking and Classification Beyond the Visible Spectrum, 2004.

[10] A. Gyaourova, G. Bebis and I. Pavlidis, Fusion of infrared and visible images for face recognition. In: European Computer Vision Conference (ECCV), 2004.

[11] G. Bebis, A. Gyaourova, S. Singh and I. Pavlidis. Face recognition by fusing thermal infrared and visible imagery. Image and Vision Computing, 24:727-742, 2006.

[12] D. A. Socolinsky and A. Selinger, A comparative analysis of face recognition performance with visible and thermal infrared imagery. In International Conference on Pattern Recognition, 4:217-222, August 2002.

[13] R. Beveridge. Evaluation of face recognition algorithms web site. http://cs.colostate.edu/evalfacerec.

[14] R.J. Micheals and T.E. Boult, Efficient evaluation of classification and recognition systems. In IEEE Computer Vision and Pattern Recognition 2001, 1:50-57, December 2001.

[15] J.R.Beveridge, K.She, B.A.Draper, and G.H.Givens, Parametric and Nonparametric Methods for the Statistical Evaluation of Human ID Algorithms. In Proceedings of the third Workshop on Empirical Evaluation Methods in Computer Vision,Kauai,HI, Dec.2001.

[16] Draper, and Geof H. Givens, A nonparametric statistical comparison of principal component and linear discriminant subspaces for face recognition. In Proceedings of the IEEE Conference on Computer Vision and Pattern Recognition, pp. 535 - 542, December 2001.

[17] http://www.equinoxsensors.com/products/HID.ht $\mathrm{ml}$.

[18] P. J. Burt and E. H. Adelson, The Laplacian pyramid as a compact image code, IEEE Trans. Common. 31:532-540, 1983

[19] A. Toet, L.J. van Ruybven and J.M. Valeton, Merging Thermal and Visual Images by a Contrast Pyramid. Optical Engineering, 28(7):789-792, 1989.

[20] S.G. Nikolov, P.R. Hill, D.R. Bull, et al, Wavelets for image fusion. In: Petrosian $\mathrm{A}$ and Meyer F (Eds.), Wavelets in signal and image analysis, Computational Imaging and Vision
Series, Kluwer Academic Publishers, Dordrecht, The Netherlands, 19: pp. 213-244, 2001.

[21] A.David and Yocky, Image merging and data fusion by means of the discrete two-dimensional wavelet transform, J.Opt. Soc. Am. A, 12(9):1834-1841, 1995.

[22] P.J. Burt and R.J. Kolczynski, Enhanced image capture through fusion. In: Proceedings of Fourth International Conference on Computer Vision, IEEE Computer Society, pp. 173 182, 1993.

[23] V.C. Klema and J. Laub, The Singular Value Decomposition: Its Eigenvector Based Algorithms. Journal of Optical Society of America, 5(10):1670-1672, 1988.

[24] Q. Tian, Y. Fainman and Z.H. Gu, Comparison of Statistical Pattern Recognition Algorithms for Hybrid Processing, Part II: Computation and Some Applications. IEEE Transactions on Automatic Control, 25(2):164-176, 1980.

[25] R. Horn and C. Johnson, Topic in Matrix Analysis. Cambridge University Press, pp. 134231, 1991. 